\title{
Overexpression of ST3Gal-I promotes migration and invasion of HCCLM3 in vitro and poor prognosis in human hepatocellular carcinoma
}

This article was published in the following Dove Press journal:

OncoTargets and Therapy

18 April 2016

Number of times this article has been viewed

\author{
Han Wu ${ }^{1, *}$ \\ Xue-Liang Shi ${ }^{1, *}$ \\ Hai-Jian Zhang ${ }^{2}$ \\ Qing-Jie Song' \\ Xiao-Bing Yang' \\ Wei-Dong $\mathrm{Hu}^{\prime}$ \\ Guang-Lin Mei' \\ Xi Chen' \\ Qin-Sheng Mao' \\ Zhong Chen' \\ 'Department of General Surgery, \\ The Affiliated Hospital of Nantong \\ University, ${ }^{2}$ Research Center of \\ Clinical Medicine, The Affiliated \\ Hospital of Nantong University, \\ Nantong, People's Republic of China \\ *These authors contributed equally \\ to this work
}

Purpose: Excessive ST3Gal-I levels predict a poor outcome for patients with several types of tumors. This study aims to investigate the role of ST3Gal-I in determining the invasive and metastatic potential of human hepatocellular carcinoma (HCC) and clinical prognosis for patients with HCC.

Methods: We compared the expression of ST3Gal-I in various HCC cell lines and in 20 pairs of tumor and peritumor tissue samples using Western blot analysis. Changes in the degree of invasiveness and migration were determined before and after small interfering RNA-induced knockdown of ST3Gal-I using a Transwell matrigel invasion assay and scratch wound assay. The correlation between ST3Gal-I expression and prognosis was determined in a large HCC patient cohort $(\mathrm{n}=273)$.

Results: ST3Gal-I expression was higher in metastatic HCCLM3 cells and tumor tissue compared with normal adjacent tissue. Following the ST3Gal-I knockdown, the invasiveness and migration of HCCLM3 cells were markedly reduced. ST3Gal-I expression in HCC correlated closely with tumor thrombus $(P<0.001)$, tumor size $(>5.0 \mathrm{~cm}, P=0.032)$, tumor node metastasis stages II-III $(P=0.002)$, and Barcelona Clinic Liver Cancer stages B-C $(P<0.001)$. Cox regression analysis demonstrated that ST3Gal-I is an independent predictor of prognosis in patients with $\mathrm{HCC}$, and related to disease-free survival (hazard ratio $=1.464, P=0.037$ ) and overall survival (hazard ratio $=1.662, P=0.012$ ).

Conclusion: ST3Gal-I might contribute to the invasiveness and metastatic nature of HCC and, thus, could be an independent predictor of recurrence and a suitable pharmaceutical target in patients with HCC.

Keywords: ST3Gal-I, hepatocellular carcinoma, sialyltransferase, prognosis, tumor progression

\section{Introduction}

Hepatocellular carcinoma (HCC) is the third most common cause of cancer death in the world and the fifth most common type of cancer. The 5-year survival rate for $\mathrm{HCC}$ after surgical resection remains low, with high rates of metastasis and recurrence serving as major obstacles to long-term survival after a curative resection has been performed. ${ }^{1}$ The low survival rate has been attributed to a delay in diagnosis and ineffective therapeutic options. ${ }^{2}$ Therefore, new diagnostic methods and therapeutic markers are needed to improve the survival of patients with this disease.

Recently, our attention has turned to the role of aberrant glycosylation in the progression and pathogenesis of various tumors. Glycosylation is an extremely important posttranslational modification of proteins. Aberrations in this process affect various
Correspondence: Zhong Chen Department of General Surgery, The Affiliated Hospital of Nantong University, 20 Xisi Road, Nantong. 22600I, People's Republic of China Tel +865138116 I00I Fax +86 5I3 8II6 I00I Email chenz9806@I63.com 
physicochemical and functional properties of proteins, such as protein stability and solubility, as well as inter- and intracellular protein transport and communication. ${ }^{3}$ A change in the level of expression of glycosyltransferases, including sialyltransferases, can alter oncogene-activated signaling pathways, thereby making significant changes in the glycan structures on glycoproteins. ${ }^{4}$ Abnormal sialylation and changes in the expression of sialyltransferase are frequently seen in many types of cancer. ${ }^{5}$ Cancer biomarkers, such as the sialylLewisa epitope (CA19-9) and sialyl-Tn epitope (CA72.4), have been used as targets for the development of anticancer immunotherapy in preclinical and clinical studies of vaccines. ${ }^{6}$ Sialyltransferase levels have been reported to correlate with tumorigenesis and progression in $\mathrm{HCC}$. Dall'Olio et $\mathrm{al}^{7}$ investigated the level of expression of the sialyltransferase ST6Gal-I and its mRNA in normal human liver tissue compared with cirrhotic tissue from patients with HCC and found that ST6Gal-I expression was the greatest in the HCC samples. This suggests that further study of sialyltransferases might provide valuable insight into the mechanisms underlying this disorder and lead to opportunities to use ST6Gal-I for identifying diagnostic, prognostic, and therapeutic approaches to HCC.

ST3Gal-I catalyzes the transfer of sialic acid in an $\alpha 2,3-$ linkage to terminal galactose residues on glycoproteins or glycolipids. Overexpression of ST3Gal-I may contribute to the initial oncogenic transformation in HCC and, therefore, might be useful for predicting cancer progression and recurrence. Using a breast cancer model, Picco et $\mathrm{al}^{8}$ reported that when crossed with PyMT mice, ST3Gal-I transgenic mice exhibited a considerable decrease in tumor latency compared with PyMT mice on an identical background, which suggests that ST3Gal-I is a tumor promoter. Sproviero et $\mathrm{al}^{9}$ found that ST3Gal-I expression is upregulated by cyclooxygenase-2 in the presence of prostaglandin E2 in the breast cancer cell line T47D. Upregulation of ST3Gal-I expression also plays an important role in the development of malignant epithelial ovarian cancer, ${ }^{10,11}$ colon carcinoma, ${ }^{12}$ bladder cancer, ${ }^{13}$ and cutaneous epithelial cancer; ${ }^{14}$ however, ST3Gal-I levels did not increase significantly in gastric cancer tissue compared with adjacent normal tissue. ${ }^{15}$ ST3Gal-I is strongly expressed in several types of tumors, but little is known about the mechanisms underlying its expression, regulation, or prognostic value in human HCC.

We investigated the relationship between ST3Gal-I expression in human $\mathrm{HCC}$ and the rate of metastasis and prognosis for the disease by analyzing its expression in $\mathrm{HCC}$ lines, resected HCC tissue specimens, and HCCLM3 cells in which ST3Gal-I activity had been silenced in vitro using small interfering RNA (siRNA). Specifically, we wanted to determine whether the overexpression of ST3Gal-I predicts a poor prognosis for patients with $\mathrm{HCC}$.

\section{Methods}

\section{Patients and specimens}

We collected 20 random HCC tissue specimens and paired adjacent tissue samples from patients who had undergone surgical resection of HCC in the Nantong University Affiliated Hospital in Nantong City, People's Republic of China, between December 2013 and July 2014 for Western blot analysis. Tumor specimens were randomly retrieved from 273 patients with HCC in the same sample bank between January 2009 and May 2011 for use in tissue microarrays. The clinicopathological characteristics of the patients from whom these samples were taken, none of whom had received any preoperative anticancer treatment, are summarized in Table S1. The study protocol, including the use of human cell lines, was approved by the Ethics Review Board of the Nantong University Affiliated Hospital. Written informed consent was obtained from all study participants.

\section{Follow-up and tumor recurrences}

Follow-up procedures are described in our previous study. ${ }^{16}$ All patients whose samples were used in the tissue microarray group were observed until March 2014 over a median of 42 months (range: 0.5-61 months). The most common causes of death among these patients were recurrence, metastasis, or liver cirrhosis. Overall survival (OS) was defined as the interval between surgery and death. Disease-free survival (DFS) was defined as the interval between surgery and recurrence.

\section{Cell culture}

The human HCC cell lines Hep3B and HepG2, provided as a gift from Cornell University, Ithaca, NY, USA, were cultured in RPMI-1640 medium supplemented with 10\% fetal bovine serum (FBS) (Hyclone, Logan, UT, USA) and penicillinstreptomycin (Thermo Fisher Scientific, Waltham, MA, USA). Human HCC cell lines HCCLM3, MHCC97H, and MHCC97L, with high and low lung metastatic potential, along with normal human hepatic L02 cells, were obtained from the General Surgery Institute of our hospital. These cells were cultured in Dulbecco minimum essential medium(DMEM; Thermo Fisher Scientific, Waltham, MA, USA) supplemented with 10\% FBS and penicillin-streptomycin at $37^{\circ} \mathrm{C}$ in $5 \% \mathrm{CO}_{2}$.

\section{Western blot analysis}

Western blot analysis was carried out as described previously. ${ }^{17}$ Briefly, cell or tissue lysates were generated and total proteins were separated using standard sodium 
dodecyl sulfate polyacrylamide gel electrophoresis and then transferred to polyvinylidene difluoride membranes. The membranes were washed and blocked before being incubated with polyclonal antibody ST3Gal-I (R\&D Systems Inc., Minneapolis, MN, USA) followed by incubation with horseradish peroxidase-conjugated secondary antibodies. The reactions were detected using an enhanced chemiluminescence assay. Glyceraldehyde-3-phosphate dehydrogenase was used as a loading control. The relative intensity of each band was determined using Image Lab 3.0 imaging methods (Bio-Rad Laboratories, Inc.; Hercules, CA, USA).

\section{siRNA-mediated ST3Gal-I silencing}

Following the manufacturer's protocol, we grew HCCLM3 cells in culture in 24-well plates or $6 \mathrm{~cm}$ dishes to a confluence of $60 \%-70 \%$ and then transfected them with siRNAs targeting human ST3Gal-I (siRNA sequence: sense 5'-GAAGAGGUAUUGAAUGCUA-3' and antisense 5'-CUUCUCCAUAACUUACGAU-3') using the siRNA transfection reagent RNAiMAX (Invitrogen). Simultaneously, they were transfected with nontargeting control siRNA as a negative control. After 72 hours of transfection, the cells were harvested for Western blot analysis.

\section{MTT assay, cell migration, and invasion assay}

Cells were aliquoted into a 96 -well plate $(5,000 / 100 \mu \mathrm{L}$ per well), incubated overnight, and then exposed to ST3Gal-I siRNA for 6 hours. The medium was then replaced with $100 \mu \mathrm{L}$ DMEM containing $10 \%$ FBS to which $10 \mu \mathrm{L}$ of CCK-8 solution (Dojindo; Kumamoto, Japan) was added at the indicated time points. The plates were then incubated an additional 2 hours. Absorbance was measured at $450 \mathrm{~nm}$ to determine the number of viable cells in each well. Each experiment was carried out three times.

Cell migration was evaluated by scratch wound assay. Briefly, $10^{6}$ HCCLM3 cells/well were plated in a 6-well plate and cultured overnight to yield a confluent monolayer. The cells were then exposed to $10 \mathrm{mg} / \mathrm{mL}$ mitomycin for 1 hour to inhibit proliferation and then wounded with a $10 \mu \mathrm{L}$ pipette tip. The remaining cells were washed twice and then cultured with DMEM supplemented with $2 \%$ FBS. Photographs were taken at 0,24 , and 48 hours. At the indicated times, migrating cells at the front of the wound were photographed and the percentage of the cleaned area at each time point was compared with the area at time 0 . Each area was measured using Image-Pro Plus version 6.2 software.

Cell invasiveness was evaluated using a Transwell matrigel invasion assay in 24-well Transwell units (Corning,
New York, NY, USA). Each well contained $10^{5}$ HCCLM3 cells suspended in DMEM. Seeded wells in the upper chamber were coated with matrigel and incubated with DMEM supplemented with $10 \% \mathrm{FBS} ; 500 \mu \mathrm{L}$ of DMEM was applied in the lower chamber. After 24 hours, invading cells on the bottom surface were fixed with $4 \%$ paraformaldehyde and counted under a light microscope after staining with crystal violet.

\section{Tissue microarray and immunohistochemistry}

Tissue microarrays were constructed as described previously. ${ }^{16}$ Immunohistochemistry was carried out as described previously. ${ }^{16}$ Briefly, after neutralizing endogenous peroxidase and retrieving antigens by microwave, we preincubated the slides with blocking serum and then incubated them overnight with mouse antihuman ST3Gal-I (R\&D Systems) polyclonal antibody. Sections were then incubated with a second antibody and exposed to horseradish peroxidase-conjugated streptavidin. Using a Leica CCD camera (DFC420), we captured photographs of three representative fields using Leica QWin Plus v3 software.

Immunohistochemical staining was assessed by two independent pathologists without the knowledge of patient characteristics. A scoring system was applied as previously described. ${ }^{17}$ The staining intensity of ST3Gal-I was scored first ( 0 : negative; 1 : weak; 2 : high) and then the percentage of positive cells was scored (1: $0 \%-25 \% ; 2$ : 26\%-50\%; 3: $51 \%-75 \% ; 4:>75 \%$ ). The final score for each sample was calculated by multiplying the staining intensity score by percentage score. Tumors with a staining index $\geq 2$ were defined as high-expression tumors.

\section{Statistical analysis}

Statistical analysis was carried out using SPSS 17.0 software (SPSS Inc., Chicago, IL, USA). Survival curves were created using the Kaplan-Meier method and compared by log-rank test. The Cox proportional hazards regression model was used for univariate and multivariate analyses, with all of the clinicopathological features used as covariates. The $\chi^{2}$ test, Fisher's exact probability, and Student's $t$-test were used for comparisons between groups. Statistical significance was established at $P<0.05$.

\section{Results}

\section{ST3Gal-I expression in HCC cell lines and tissue samples}

ST3Gal-I expression was determined in several HCC cell lines (HCCLM3, MHCC97H, MHCC97L, HepG2, Hep3B, and L02), each with a different metastatic potential. We found 
that ST3Gal-I expression increased with metastatic potential: it was significantly higher in HCCLM3 and MHCC97H cells compared with MHCC97L, HepG2, and Hep3B cells $(P<0.05$; Figure 1A) and in HCC tissue $(n=20)$ compared with paired peritumor tissue $(P<0.05$; Figure $1 \mathrm{~B})$.

\section{ST3Gal-I knockdown attenuated proliferation and reduced migration and invasion of $\mathrm{HCC}$ cells}

To further examine the functional role of ST3Gal-I in the HCC lines, we used siRNA to silence ST3Gal-I expression in HCCLM3 cells. Successful knockdown of ST3Gal-I expression was confirmed using Western blot analysis $(P=0.01$, Figure 2A). Down-regulation of ST3Gal-I resulted in a significant suppression of cell proliferation within 72 hours following transfection $(P<0.001$; Figure 2B). The Transwell matrigel invasion assay indicated that silencing of ST3Gal-I expression also significantly reduced the invasiveness of HCCLM3 cells in vitro (number of invaded cells [controls vs siRNA]: 137 vs 71, respectively; $P=0.012$ ) (Figure 2C). Microscopic examinations at 24 and 48 hours following the scratch wound assay revealed a decreased migration of HCCLM3 cells in the siRNA group compared with controls $(P<0.001$; Figure 2D).

\section{Patient clinicopathological profiles}

The median follow-up was 42.0 months (range: 0.5-61.0 months; standard deviation: 18 months). DFS and OS rates at 1,3 , and 5 years posthepatectomy were $79.4 \%$ and $88.7 \%$, $56.7 \%$ and $70.1 \%$, and $47.6 \%$ and $59.2 \%$, respectively. At the last follow-up visit, $133(48.72 \%)$ patients were confirmed to have had a relapse characterized by an intrahepatic recurrence $(n=89)$, extrahepatic metastasis $(n=32)$, or both $(n=12)$. Additionally, 105 patients $(38.46 \%)$ had died of recurrence $(n=74)$ or a cirrhosis-related complication without recurrence $(n=31)$.

\section{ST3Gal-I expression and patient outcomes}

Immunohistochemical staining showed that ST3Gal-I was present mainly in the tumor cell membrane, cytoplasm, or both in a focal or scattered pattern (Figure 3A). Of the 273 patients with $\mathrm{HCC}, 252$ (92.3\%) were positive for ST3Gal-I, with 137 (50.2\%) considered high-expression patients and $136(49.8 \%)$ considered low-expression patients. Only 21 (7.7\%) were negative for ST3Gal-I expression. Most of the peritumor tissue samples were negative for ST3Gal-I or demonstrated low levels of ST3Gal-I expression.

Univariate analysis indicated that compared with patients expressing low levels of ST3Gal-I, high-expression patients had a significantly worse DFS (43.93 vs 34.51 months, respectively; $P=0.005$ ) and $\mathrm{OS}$ (51.6 vs 42.06 months, respectively; $P=0.001$ ) (Figure 3B). Multivariate analysis suggested that the level of ST3Gal-I expression was an independent predictor of OS $(P=0.012 ; \mathrm{HR}=1.662)($ Table 1$)$ and DFS $(P=0.037 ; \mathrm{HR}=1.464)$
A
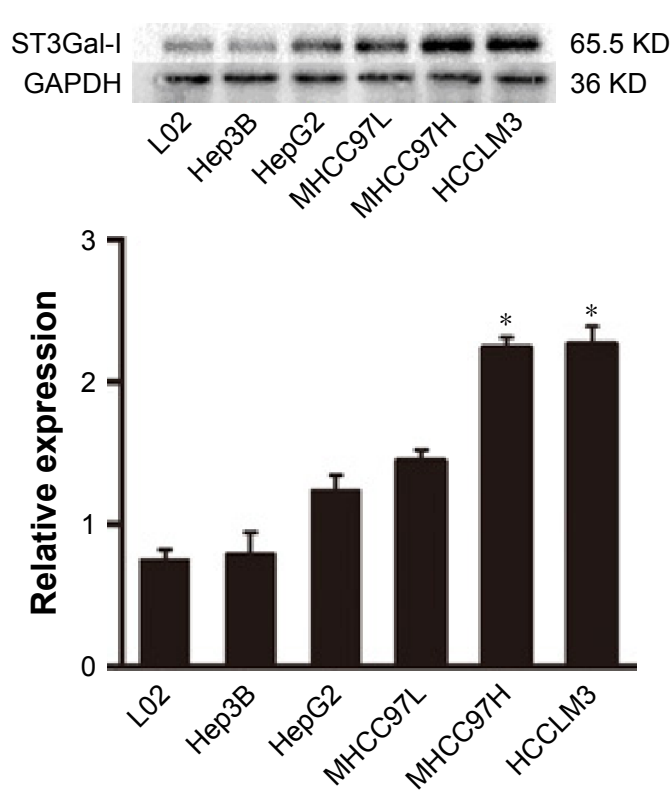

B
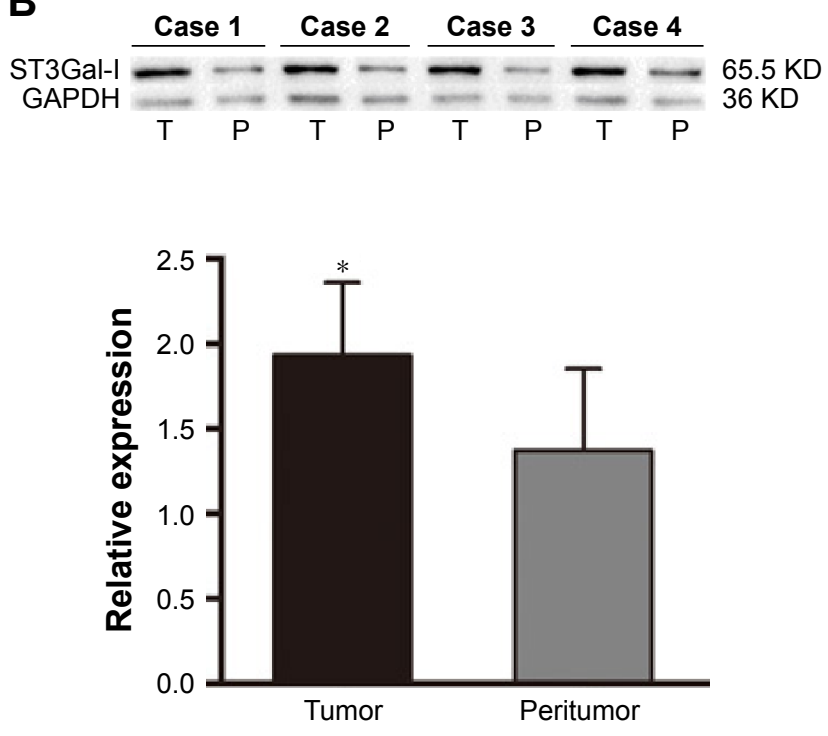

Figure I Expression of ST3Gal-I in HCC cell lines and HCC tissue samples.

Notes: Using Western blot analysis, we detected ST3Gal-I expression in hepatic and HCC cell lines (A); expression of ST3Gal-I was increased in 20 tumor tissues compared with peritumor tissues $(\mathbf{B}), * P<0.05$.

Abbreviations: HCC, hepatocellular carcinoma; GADPH, glyceraldehyde 3-phosphate dehydrogenase. 
A
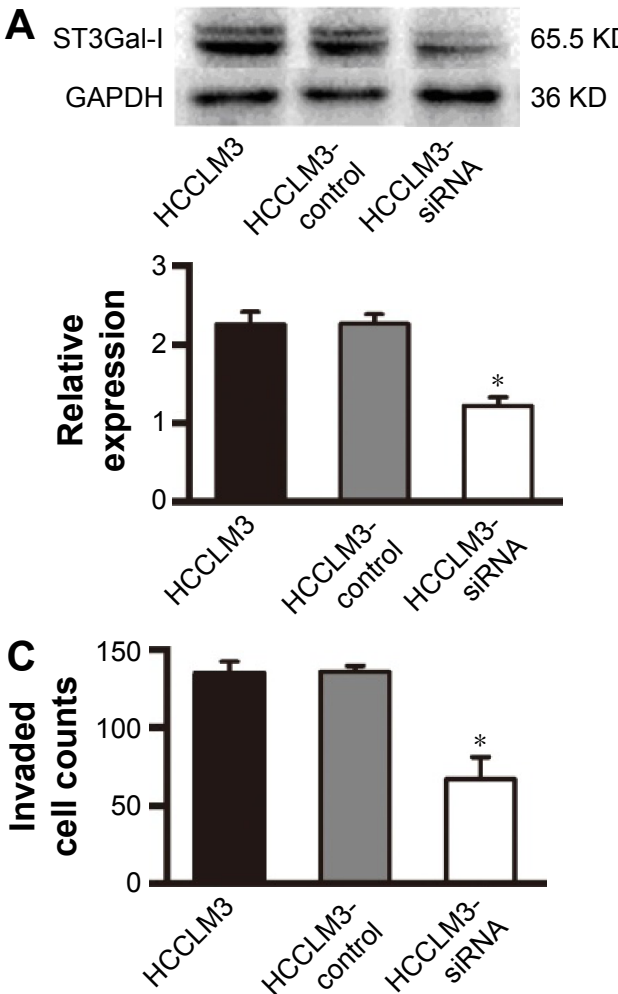

D
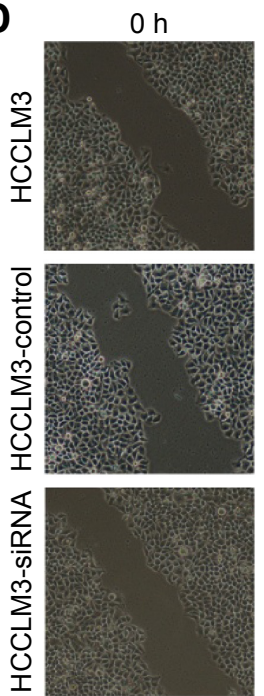

$24 \mathrm{~h}$
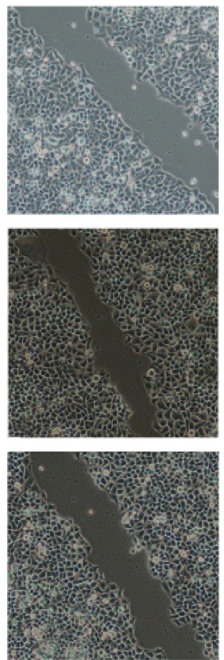

B
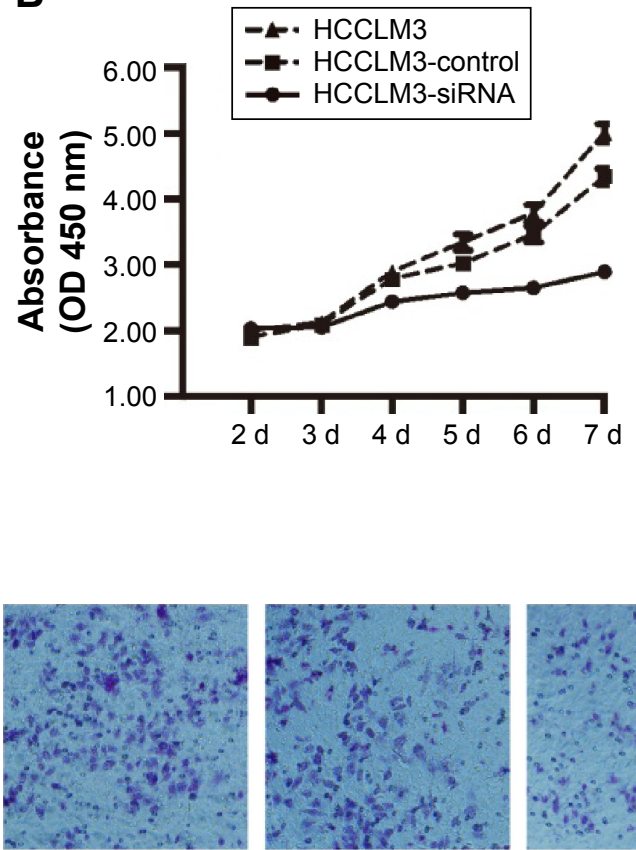

HCCLM3

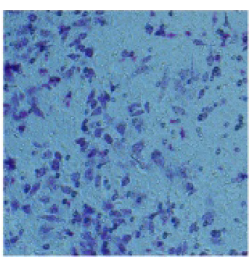

HCCLM3-control

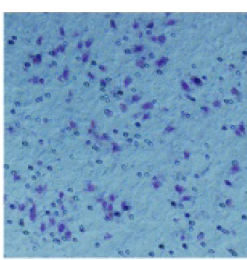

HCCLM3-SIRNA

$48 \mathrm{~h}$
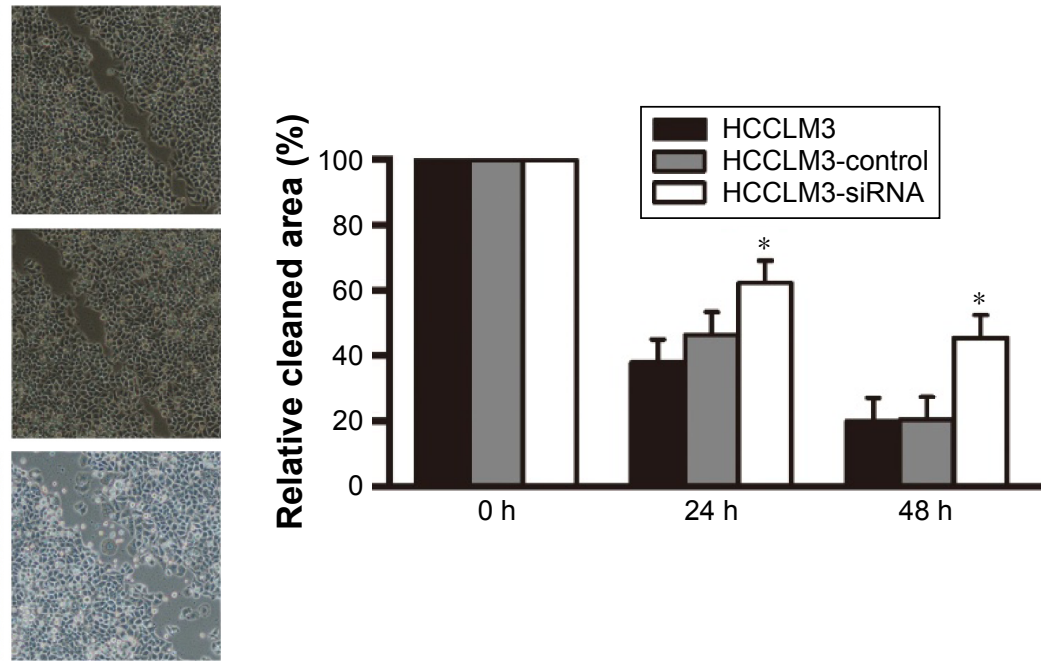

Figure 2 Function analysis after ST3Gal-I siRNA in HCCLM3.

Notes: siRNA efficiently down-regulated the expression of ST3Gal-I protein in HCCLM3 cells (A). Cell proliferation was detected by MTT assay. Mean \pm SD was calculated for data derived from individual experiments with three replicate assays (B). After knockdown of ST3Gal-I expression, cellular invasion (C) and migration (D) of HCCLM3 was reduced compared with controls. Magnification: $\times 200$ (C) and $\times 100$ (D). $* P<0.005$.

Abbreviations: siRNA, small interfering RNA; SD, standard deviation; GADPH, glyceraldehyde 3-phosphate dehydrogenase; h, hours; d, day; OD, optical density; MTT, 3-(4, 5-dimethyl-2-thiazolyl)-2,5-diphenyl-2-H-tetrazolium bromide.

(Table 2). It also revealed that liver cirrhosis $(P=0.048$, $\mathrm{HR}=1.653)$, tumor size $(P<0.001, \mathrm{HR}=2.204)$, and Barcelona Clinic Liver Cancer (BCLC) stages B and C $(P=0.005$, $\mathrm{HR}=1.665$ ) were associated with DFS (Table 2) and tumor size $(P<0.001, \mathrm{HR}=3.574)$. Survival analysis revealed that BCLC stages $\mathrm{B}$ and $\mathrm{C}(P=0.012, \mathrm{HR}=1.657)$ were associated with $\mathrm{OS}$ when integrated with ST3Gal-I expression (Table 1).
Because the BCLC stage represents different stages of HCC progression, ${ }^{18}$ a subgroup analysis was carried out to clarify the function of ST3Gal-I expression at different stages of the disease. A univariate analysis revealed that high levels of ST3Gal-I are closely associated with accelerated recurrence during the early stages of tumor development $(P=0.027)$ (Figure 3C; Table 2 ) whereas multivariate analysis 

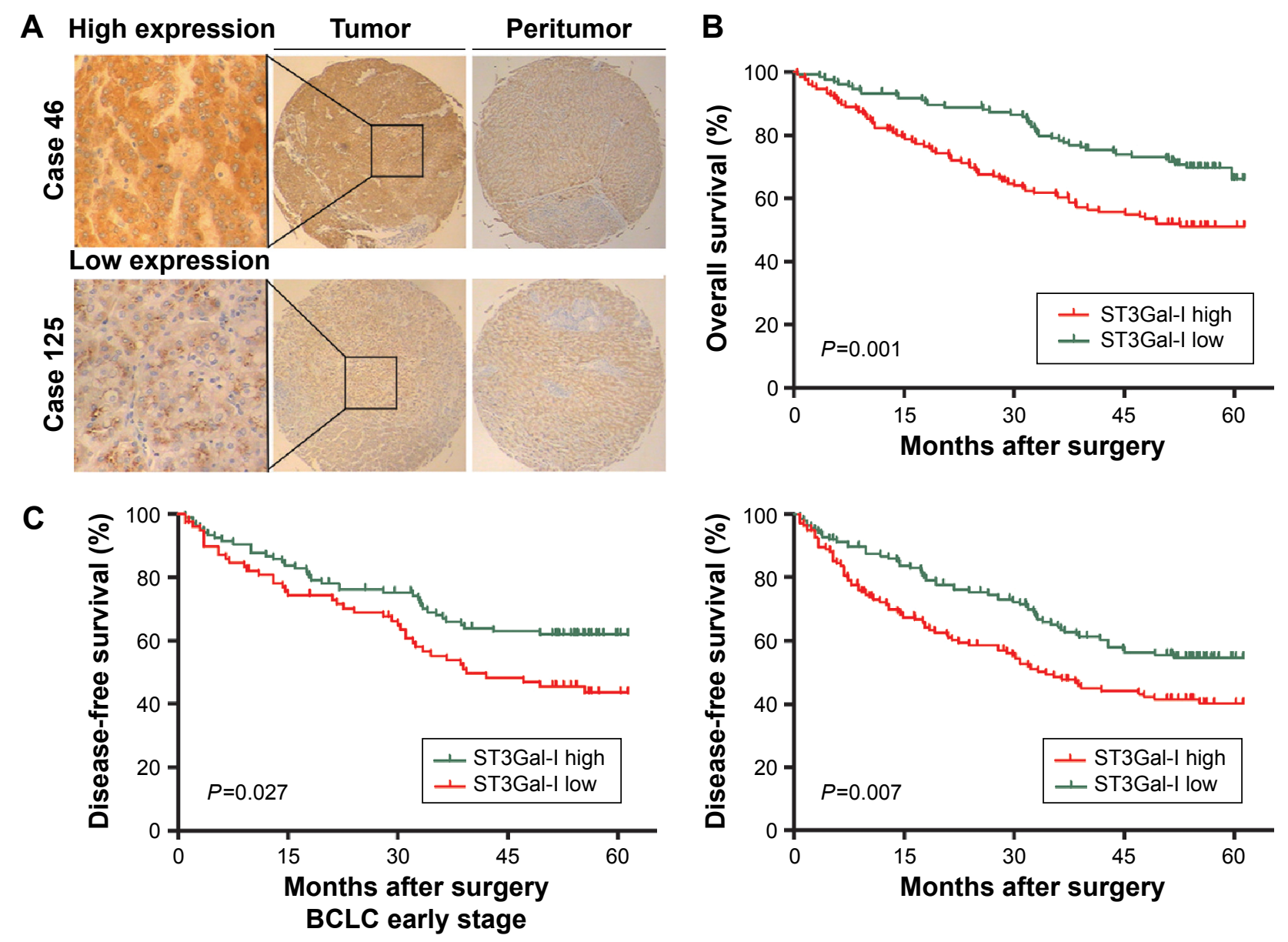

Figure 3 ST3Gal-I expression in tissue microarrays and Kaplan-Meier curves of survival differences between HCC patients.

Notes: Representative images of ST3Gal-I in HCC tumor tissues microarrays (A), magnification: $\times 40, \times 200$, respectively. Kaplan-Meier analysis of DFS and OS for ST3Gal-I expression in 273 cases (B) and Kaplan-Meier analysis of DFS of ST3Gal-I in early stage cases (C).

Abbreviations: HCC, hepatocellular carcinoma; OS, overall survival; DFS, disease-free survival; BCLC, Barcelona Clinic Liver Cancer.

Table I Univariate and multivariate analyses of prognostic factors associated with survival

\begin{tabular}{|c|c|c|c|c|}
\hline \multirow[t]{3}{*}{ Variables } & \multicolumn{4}{|l|}{ OS } \\
\hline & \multirow{2}{*}{$\frac{\text { Univariate }}{P \text {-value }}$} & \multicolumn{3}{|c|}{ Multivariate } \\
\hline & & $P$-value & HR & $95 \% \mathrm{Cl}$ \\
\hline AFP level, ng/mL ( $\leq 20$ vs $>20)$ & 0.018 & NS & & \\
\hline$\gamma$-GT, U/L $(\leq 54$ vs $>54)$ & 0.002 & NS & & \\
\hline HBV & 0.212 & NA & & \\
\hline Liver cirrhosis (no vs yes) & 0.687 & NA & & \\
\hline Tumor differentiation (I-II vs III-IV) & 0.05 & NS & & \\
\hline Tumor size, $\mathrm{cm}(\leq 5 \mathrm{vs}>5)$ & $<0.001$ & $<0.001$ & 3.574 & $2.24 I-5.286$ \\
\hline Tumor number (single vs multiple) & 0.552 & NA & & \\
\hline Encapsulation (complete vs none) & 0.214 & NA & & \\
\hline Tumor thrombus (no vs yes) & 0.001 & NS & & \\
\hline TNM stage (I vs II-III ) & $<0.001$ & NS & & \\
\hline$B C L C$ stage ( $A$ vs $B$ and $C)$ & 0.001 & 0.012 & 1.657 & $1.121-2.439$ \\
\hline ST3Gal-I (low vs high) & 0.001 & 0.012 & 1.662 & $1.123-2.477$ \\
\hline
\end{tabular}

Notes: Univariate analysis was calculated by the Kaplan-Meier method (the log-rank test). Multivariate analysis was done using the Cox multivariate proportional hazards regression model with stepwise manner (forward, likelihood ratio).

Abbreviations: AFP, alpha-fetoprotein; $\gamma$-GT, $\gamma$-glutamyl transferase; TNM stage, tumor node metastasis stage; BCLC stage, Barcelona Clinic Liver Cancer stage; OS, overall survival; $\mathrm{HR}$, hazard ratio; $\mathrm{Cl}$, confidence interval; NS, not significant; NA, not applicable; HBV, Hepatitis B Virus. 
Table 2 Univariate and multivariate analyses of ST3Gal-I associated with early and late recurrence

\begin{tabular}{|c|c|c|c|c|}
\hline \multirow[t]{3}{*}{ Variables } & \multicolumn{4}{|l|}{ DFS } \\
\hline & \multirow{2}{*}{$\frac{\text { Univariate }}{P \text {-value }}$} & \multicolumn{3}{|c|}{ Multivariate } \\
\hline & & $P$-value & HR & $95 \% \mathrm{Cl}$ \\
\hline \multicolumn{5}{|l|}{ Early stage } \\
\hline AFP level, $\mathrm{ng} / \mathrm{mL}$ ( $\leq 20$ vs $>20$ ) & 0.203 & NA & & \\
\hline Tumor size, $\mathrm{cm}(\leq 5 \mathrm{vs}>5)$ & $<0.001$ & $<0.001$ & 2.452 & $1.569-3.833$ \\
\hline Tumor number (single vs multiple) & 0.476 & NA & & \\
\hline Encapsulation (complete vs none) & 0.715 & NA & & \\
\hline Tumor differentiation (I-II vs III-IV) & 0.612 & NA & & \\
\hline ST3Gal-I (low vs high) & 0.027 & 0.051 & & \\
\hline \multicolumn{5}{|l|}{ Tissue microarray (TMA) assay } \\
\hline AFP level, $\mathrm{ng} / \mathrm{mL}(\leq 20$ vs $>20)$ & 0.034 & NS & & \\
\hline$\gamma$-GT, U/L $(\leq 54$ vs $>54)$ & 0.002 & NS & & \\
\hline $\mathrm{HBV}$ & 0.352 & NA & & \\
\hline Liver cirrhosis (no vs yes) & 0.041 & 0.048 & 1.653 & $1.021-2.664$ \\
\hline Tumor differentiation (I-II vs III-IV) & 0.266 & NA & & \\
\hline Tumor size, $\mathrm{cm}(\leq 5 \mathrm{vs}>5)$ & $<0.001$ & $<0.001$ & 2.204 & $1.564-3.127$ \\
\hline Tumor number (single vs multiple) & 0.357 & NA & & \\
\hline Encapsulation (complete vs none) & 0.165 & NA & & \\
\hline Tumor thrombus (no vs yes) & 0.001 & NS & & \\
\hline TNM stage (I vs II-III) & $<0.001$ & NS & & \\
\hline BCLC stage (A vs B-C) & 0.001 & 0.005 & 1.665 & I.17I-2.357 \\
\hline ST3Gal-I (low vs high) & 0.007 & 0.037 & 1.464 & $1.021-2.093$ \\
\hline
\end{tabular}

Notes: Univariate analysis was calculated by the Kaplan-Meier method (the log-rank test). Multivariate analysis was done using the Cox multivariate proportional hazards regression model with stepwise manner (forward, likelihood ratio).

Abbreviations: AFP, alpha-fetoprotein; $\gamma$-GT, $\gamma$-glutamyl transferase; TNM stage, tumor node metastasis stage; BCLC stage, Barcelona Clinic Liver Cancer stage; DFS, diseasefree survival; HR, hazard ratio; $\mathrm{Cl}$, confidence interval; NS, not significant; NA, not applicable; HBV, Hepatitis B Virus.

indicated that ST3Gal-I expression is only of borderline significance $(P=0.051)$.

\section{Correlation of ST3Gal-I expression and clinicopathological characteristics}

To evaluate the relationship between ST3Gal-I and tumor biology, we compared clinicopathological features with ST3Gal-I expression. We found that upregulation of ST3Gal-I protein levels correlated significantly with HCC aggressiveness, for example, tumor thrombus $(P<0.001)$, tumor size ( $>5 \mathrm{~cm}, P=0.032$ ), and advanced tumor stage (BCLC stages $\mathrm{B}$ and $\mathrm{C}, P<0.001$; tumor node metastasis stage II-III, $P=0.002$ ) (Table 3).

\section{Discussion}

In the present study, we explored the expression of ST3Gal-I in human HCC cell lines with high and low metastatic potential and resected cancer specimens. We determined that ST3Gal-I expression correlates significantly with malignant tumor behavior in HCC. Using high-throughput tissue microarrays to examine the prognostic potential of ST3Gal-I expression in tumor tissue samples surgically resected from patients with $\mathrm{HCC}$, we found a significant relationship between ST3Gal-I expression and both a poor prognosis and early-stage BCLC, which suggests that upregulation of ST3Gal-I may represent an early event in tumor development.

Recently, aberrant sialyltransferase expression was found to be related to malignant behavior in many types of cancer and regarded as a poor prognostic factor. ${ }^{11,15}$ However, reports of the prognostic potential of sialyltransferases in patients with tumors have been few and with inconsistent results. The highest levels of ST6Gal-I expression have been seen in grade $2 \mathrm{HCC}$ tumors, $\mathrm{HCC}$ without preexisting cirrhosis, and male patients with HCC. ${ }^{7}$ Poon et al, ${ }^{19}$ however, associated a decrease in tumor ST6Gal-I activity with a shorter OS in patients with HCC and found a negative correlation between tumor ST6Gal-I activity and serum levels of monosialylated alpha-fetoprotein. By contrast, Patani et $\mathrm{a}^{20}$ associated high levels of ST6GalNAc-I expression in breast cancer tissue with a better prognosis.

Increasing amounts of evidence have demonstrated that ST3Gal-I plays an important role in key pathophysiological events during various stages of tumor progression, including invasion and metastasis. Wang et $\mathrm{al}^{11}$ suggested that altered expression of sialyltransferase ST3Gal-I mRNA is 
Table 3 Correlation of clinicopathologic characteristics with ST3Gal-I expression

\begin{tabular}{|c|c|c|c|c|c|}
\hline \multirow[t]{2}{*}{ Characteristics } & \multicolumn{5}{|c|}{ ST3Gal-I $(n=273)$} \\
\hline & Total & Low & High & $x^{2}$ & $P$-value \\
\hline Age (years) & & & & 2.667 & 0.102 \\
\hline$\leq 50$ & 133 & 73 & 60 & & \\
\hline$>50$ & 140 & 63 & 77 & & \\
\hline Sex & & & & 0.469 & 0.493 \\
\hline Female & 44 & 24 & 20 & & \\
\hline Male & 229 & 112 & 117 & & \\
\hline $\mathrm{HBsAg}$ & & & & 0.225 & 0.894 \\
\hline No & 46 & 26 & 20 & & \\
\hline Yes & 227 & 113 & 114 & & \\
\hline AFP (ng/mL) & & & & 1.45 & 0.228 \\
\hline$\leq 20$ & 102 & 46 & 56 & & \\
\hline$>20$ & 171 & 90 & 81 & & \\
\hline$\gamma-\mathrm{GT}(\mathrm{U} / \mathrm{L})$ & & & & 1.322 & 0.25 \\
\hline$\leq 54$ & 123 & 66 & 57 & & \\
\hline$>54$ & 150 & 70 & 80 & & \\
\hline Tumor differentiation & & & & 0.001 & 0.972 \\
\hline I-II & 205 & 102 & 103 & & \\
\hline III-IV & 68 & 34 & 34 & & \\
\hline Tumor size $(\mathrm{cm})$ & & & & 4.579 & 0.032 \\
\hline$\leq 5$ & 182 & 99 & 83 & & \\
\hline$>5$ & 91 & 37 & 54 & & \\
\hline Tumor number & & & & 0.023 & 0.879 \\
\hline Single & 236 & 118 & 118 & & \\
\hline Multiple & 37 & 18 & 19 & & \\
\hline Encapsulation & & & & 0.092 & 0.762 \\
\hline None & 138 & 70 & 68 & & \\
\hline Complete & 135 & 66 & 69 & & \\
\hline Tumor thrombus & & & & 12.272 & $<0.001$ \\
\hline No & 199 & 112 & 87 & & \\
\hline Yes & 74 & 24 & 50 & & \\
\hline TNM stage & & & & 9.584 & 0.002 \\
\hline 1 & 123 & 74 & 49 & & \\
\hline II-III & 150 & 62 & 88 & & \\
\hline BCLC stage & & & & 12.846 & $<0.001$ \\
\hline A & 185 & 106 & 79 & & \\
\hline$B$ and $C$ & 88 & 30 & 58 & & \\
\hline
\end{tabular}

Note: Statistical analyses were done by the $\chi^{2}$ test.

Abbreviations: AFP, alpha-fetoprotein; $\mathrm{HBs} A g$, hepatitis $B$ surface antigen; $\gamma-G T$, $\gamma$-glutamyl transferase; TNM stage, tumor node metastasis stage; BCLC stage, Barcelona Clinic Liver Cancer stage.

important in malignant ovarian cancer. ST3Gal-I expression was significantly increased in colorectal cancer specimens compared with specimens of normal mucosal tissue and specimens obtained in the presence of local lymph invasion. ${ }^{21}$ Our results were consistent with these reports. Furthermore, we demonstrated that silencing of ST3Gal-I expression significantly reduces the invasive and migratory characteristics of HCCLM3 cells in vitro. Nevertheless, Zhang et al ${ }^{22}$ reported that ST3Gal-I is down-regulated in the HCC cell lines HepG2 and SMMC-7721 compared with the normal hepatic cell line L02. It has also been reported that there is no significant difference in ST3Gal-I transcription levels in MHCC97H versus MHCC97L cells. ${ }^{23}$ Conflicting information has also been reported concerning the role of ST3Gal-I expression in breast cancer. Down-regulation of ST3Gal-I has been associated with breast cancer, ${ }^{24}$ but Picco et al ${ }^{8}$ found that overexpression of ST3Gal-I promotes mammary tumorigenesis. These findings suggest that further study is needed to determine the role of different patterns of ST3Gal-I expression in different types of tumors. We also found that upregulation of ST3Gal-I significantly correlates with HCC aggression, including tumor thrombus, large tumor size, and advanced tumor stage. These results strongly indicate that ST3Gal-I is involved in HCC cell migration or metastasis and is related to a poor prognosis for patients with HCC.

It is now well established that malignant tumor behavior may result from the upregulation of sialyltransferase activity during tumor biosynthesis; unfortunately, the precise molecular mechanisms underlying this process remain unknown. Solatycka et $\mathrm{al}^{24}$ demonstrated that changes in the structure of O-linked oligosaccharides that result in the appearance of $T$ antigens are at least partially associated with the upregulation of mucin1, which down-regulates the expression of ST3Gal-I in T47D cells. It is well known that the tumor microenvironment and inflammation play vital roles in liver cancer. It was suggested that the increased sialylation on the liver membrane of Engelbreth-Holm-Swarm-bearing mice is associated with upregulation of sialyltransferase expression, which plays a role in tumor-induced liver inflammation, and that ST3Gal-I is upregulated in the liver of tumor-bearing mice compared with non-tumor-bearing controls. ${ }^{25}$ Recently, it was reported that ST6Gal-I can regulate the invasiveness and chemosensitivity of HCC tumors by regulating the activity of the PI3K/Akt pathway in MHCC $97 \mathrm{H}$ and MHCC97L cells..$^{23}$ These findings suggest that ST3Gal-I can serve as a target for the development of molecular therapeutic agents against HCC.

Thus far, sialyltransferase inhibitors have been used to study the role of sialylation in cancer progression and metastasis. They may serve as potent antimetastatic agents in future therapeutic applications. The sialyltransferase inhibitor KI-8110 efficiently down-regulated the pulmonary metastatic potential of murine NL-17 colon adenocarcinoma cells ${ }^{26}$ by inhibiting the platelet-derived growth factordependent growth of cancer cells. ${ }^{27}$ Soyasaponin I, the first CMP-Neu5Ac competitive inhibitor of ST3Gal-I in vivo, ${ }^{28}$ enhanced cell adhesion and decreased the migratory and pulmonary metastatic potential of B16F10 melanoma cells in mice. ${ }^{29}$ Additionally, AL10 inhibited the adhesion, migration, and invasiveness of human lung cancer cells overexpressing 
ST3Gal-I, A549, and CL1.5. It was also associated with reduced integrin signaling and significant suppression of experimentally-induced lung metastasis. ${ }^{30}$ The findings of our study and the research described thus far suggest that sialyltransferase inhibitors can reduce the invasive behavior of tumor cells and, thus, might serve as a target for developing new treatment strategies for patients with HCC.

In conclusion, the present study has associated ST3Gal-I expression with a worse prognosis and increased tumor invasiveness in HCC. We propose, therefore, that ST3Gal-I may be an appropriate target for developing molecular therapeutic approaches to $\mathrm{HCC}$.

\section{Acknowledgments}

This study was funded by the National Natural Science Foundation of China (Grant 81401988), the Special Project for Clinical Medicine of Jiangsu Province Department of Science and Technology (Grant BI2014060), and the Project of Nantong Science and Technology Bureau, Jiangsu Province, China (Grant MS22015116). No benefits in any form have been received or will be received from a commercial party related directly or indirectly to the subject of this article.

\section{Disclosure}

The authors report no conflicts of interest in this work.

\section{References}

1. Fitzmorris P, Shoreibah M, Anand BS, Singal AK. Management of hepatocellular carcinoma. J Cancer Res Clin Oncol. 2015;141(5):861-876.

2. Aref AM, Hoa NT, Ge L, et al. HCA519/TPX2: a potential T-cell tumor-associated antigen for human hepatocellular carcinoma. Onco Targets Ther. 2014;13(7):1061-1070.

3. Meany DL, Chan DW. Aberrant glycosylation associated with enzymes as cancer biomarkers. Clin Proteomics. 2011;8(1):7.

4. Remmers N, Anderson JM, Linde EM, et al. Aberrant expression of mucin core proteins and o-linked glycans associated with progression of pancreatic cancer. Clin Cancer Res. 2013;19(8):1981-1993.

5. Harduin-Lepers A, Krzewinski-Recchi MA, Colomb F, Foulquier F, Groux-Degroote S, Delannoy P. Sialyltransferases functions in cancers. Front Biosci (Elite Ed). 2012;1(4):499-515.

6. Reis CA, Osorio H, Silva L, Gomes C, David L. Alterations in glycosylation as biomarkers for cancer detection. J Clin Pathol. 2010;63(4): 322-329.

7. Dall'Olio F, Chiricolo M, D'Errico A, et al. Expression of betagalactoside alpha2,6 sialyltransferase and of alpha2,6-sialylated glycoconjugates in normal human liver, hepatocarcinoma, and cirrhosis. Glycobiology. 2004;14(1):39-49.

8. Picco G, Julien S, Brockhausen I, et al. Over-expression of ST3Gal-I promotes mammary tumorigenesis. Glycobiology. 2010;20(10): $1241-1250$.

9. Sproviero D, Julien S, Burford B, Taylor-Papadimitriou J, Burchell JM. Cyclooxygenase-2 enzyme induces the expression of the alpha2,3-sialyltransferase-3 (ST3Gal-I) in breast cancer. J Biol Chem. 2012;287(53):44490-44497.

10. Wang PH, Li YF, Juang CM, et al. Expression of sialyltransferase family members in cervix squamous cell carcinoma correlates with lymph node metastasis. Gynecol Oncol. 2002;86(1):45-52.
11. Wang PH, Lee WL, Juang CM, et al. Altered mRNA expressions of sialyltransferases in ovarian cancers. Gynecol Oncol. 2005;99(3):631-639.

12. Kudo T, Ikehara Y, Togayachi A, et al. Up-regulation of a set of glycosyltransferase genes in human colorectal cancer. Lab Invest. 1998;78(7): 797-811.

13. Videira PA, Correia M, Malagolini N, et al. ST3Gal.I sialyltransferase relevance in bladder cancer tissues and cell lines. BMC Cancer. 2009; 9:357.

14. Ferreira SA, Vasconcelos JL, Silva RC, et al. Expression patterns of alpha2,3-sialyltransferase I and alpha2,6-sialyltransferase I in human cutaneous epithelial lesions. Eur J Histochem. 2013;57(1):e7.

15. Jun L, Yuanshu W, Yanying X, et al. Altered mRNA expressions of sialyltransferases in human gastric cancer tissues. Med Oncol. 2012; 29(1):84-90.

16. Wu H, Chen P, Liao R, et al. Overexpression of galectin-1 is associated with poor prognosis in human hepatocellular carcinoma following resection. J Gastroenterol Hepatol. 2012;27(8):1312-1319.

17. Yi Y, Wu H, Gao Q, et al. Interferon regulatory factor (IRF)-1 and IRF-2 are associated with prognosis and tumor invasion in HCC. Ann Surg Oncol. 2013;20(1):267-276.

18. Xu L, Gao H, Huang J, et al. Antiviral therapy in the improvement of survival of patients with hepatitis B virus-related hepatocellular carcinoma treated with sorafenib. J Gastroenterol Hepatol. 2015;30(6): 1032-1039.

19. Poon TC, Chiu CH, Lai PB, et al. Correlation and prognostic significance of beta-galactoside alpha-2,6-sialyltransferase and serum monosialylated alpha-fetoprotein in hepatocellular carcinoma. World J Gastroenterol. 2005;11(42):6701-6706.

20. Patani N, Jiang W, Mokbel K. Prognostic utility of glycosyltransferase expression in breast cancer. Cancer Genomics Proteomics. 2008;5(6): 333-340.

21. Schneider F, Kemmner W, Haensch W, et al. Overexpression of sialyltransferase CMP-sialic acid:Galbeta1,3GalNAc-R alpha6Sialyltransferase is related to poor patient survival in human colorectal carcinomas. Cancer Res. 2001;61(11):4605-4611.

22. Zhang Y, Zhao W, Zhao Y, He Q. Expression of ST3Gal, ST6Gal, ST6GalNAc and ST8Sia in human hepatic carcinoma cell lines, HepG-2 and SMMC-7721 and normal hepatic cell line, L-02. Glycoconj J. 2015; 32(1-2):39-47.

23. Zhao Y, Li Y, Ma H, et al. Modification of sialylation mediates the invasive properties and chemosensitivity of human hepatocellular carcinoma. Mol Cell Proteomics. 2014;13(2):520-536.

24. Solatycka A, Owczarek T, Piller F, et al. MUC1 in human and murine mammary carcinoma cells decreases the expression of core 2 beta1,6$\mathrm{N}$-acetylglucosaminyltransferase and beta-galactoside alpha2,3sialyltransferase. Glycobiology. 2012;22(8):1042-1054.

25. Lee A, Chick JM, Kolarich D, et al. Liver membrane proteome glycosylation changes in mice bearing an extra-hepatic tumor. Mol Cell Proteomics. 2011;10(9):M900538MCP900200.

26. Kijima-Suda I, Miyamoto Y, Toyoshima S, Itoh M, Osawa T. Inhibition of experimental pulmonary metastasis of mouse colon adenocarcinoma 26 sublines by a sialic acid: nucleoside conjugate having sialyltransferase inhibiting activity. Cancer Res. 1986;46(2):858-862.

27. Kijima-Suda I, Miyazawa T, Itoh M, Toyoshima S, Osawa T. Possible mechanism of inhibition of experimental pulmonary metastasis of mouse colon adenocarcinoma 26 sublines by a sialic acid: nucleoside conjugate. Cancer Res. 1988;48(13):3728-3732.

28. Wu CY, Hsu CC, Chen ST, Tsai YC. Soyasaponin I, a potent and specific sialyltransferase inhibitor. Biochem Biophys Res Commun. 2001;284(2): 466-469.

29. Chang WW, Yu CY, Lin TW, Wang PH, Tsai YC. Soyasaponin I decreases the expression of alpha2,3-linked sialic acid on the cell surface and suppresses the metastatic potential of B16F10 melanoma cells. Biochem Biophys Res Commun. 2006;341(2):614-619.

30. Chiang CH, Wang CH, Chang HC, More SV, Li WS, Hung WC. A novel sialyltransferase inhibitor AL10 suppresses invasion and metastasis of lung cancer cells by inhibiting integrin-mediated signaling. $J$ Cell Physiol. 2010;223(2):492-499. 


\section{Supplementary material}

Table SI Clinicopathologic characteristics of the HCC patients

\begin{tabular}{|c|c|c|c|}
\hline Indexes & Characteristics & Number & $\%$ \\
\hline \multirow[t]{2}{*}{ Age (years) } & $\leq 50$ & 133 & 48.7 \\
\hline & $>50$ & 140 & 51.3 \\
\hline \multirow[t]{2}{*}{ Sex } & Female & 43 & 15.8 \\
\hline & Male & 230 & 84.2 \\
\hline \multirow[t]{2}{*}{ Liver cirrhosis } & No & 240 & 87.9 \\
\hline & Yes & 33 & 12.1 \\
\hline \multirow[t]{2}{*}{$\mathrm{HBsAg}$} & Negative & 46 & 16.8 \\
\hline & Positive & 227 & 83.2 \\
\hline \multirow[t]{2}{*}{$\mathrm{HCV}$} & Negative & 269 & 98.5 \\
\hline & Positive & 4 & 1.5 \\
\hline \multirow{2}{*}{$\gamma-\mathrm{GT}(\mathrm{U} / \mathrm{L})$} & $\leq 54$ & 123 & 45.1 \\
\hline & $>54$ & 150 & 54.9 \\
\hline \multirow[t]{2}{*}{$\operatorname{AFP}(\mathrm{ng} / \mathrm{mL})$} & $\leq 20$ & $17 \mid$ & 62.6 \\
\hline & $>20$ & 102 & 37.4 \\
\hline \multirow{2}{*}{ Child-Pugh score } & A & 254 & 93.0 \\
\hline & B & 19 & 7.0 \\
\hline \multirow[t]{2}{*}{ Tumor differentiation } & I-II & 205 & 75.1 \\
\hline & III-IV & 68 & 24.9 \\
\hline \multirow[t]{2}{*}{ Tumor size $(\mathrm{cm})$} & $\leq 5$ & 182 & 66.7 \\
\hline & $>5$ & 91 & 33.3 \\
\hline \multirow[t]{2}{*}{ Tumor number } & Single & 236 & 86.4 \\
\hline & Multiple & 37 & 13.6 \\
\hline \multirow[t]{2}{*}{ Tumor encapsulation } & Complete & 138 & 50.5 \\
\hline & None & 135 & 49.5 \\
\hline \multirow[t]{2}{*}{ Tumor thrombus } & No & 199 & 72.9 \\
\hline & Yes & 74 & 27.1 \\
\hline \multirow[t]{2}{*}{ TNM stage } & I & 123 & 45.1 \\
\hline & II-III & 150 & 54.9 \\
\hline \multirow[t]{2}{*}{$\mathrm{BCLC}$ stage } & A & 185 & 67.8 \\
\hline & $B$ and $C$ & 88 & 32.2 \\
\hline
\end{tabular}

Abbreviations: HCC, hepatocellular carcinoma; AFP, alpha-fetoprotein; $\gamma$-GT, $\gamma$-glutamyl transferase; $\mathrm{HBsAg}$, hepatitis $B$ surface antigen; $\mathrm{HCV}$, hepatitis $\mathrm{C}$ virus; TNM stage, tumor node metastasis stage; BCLC, Barcelona Clinic Liver Cancer.

\section{Publish your work in this journal}

OncoTargets and Therapy is an international, peer-reviewed, open access journal focusing on the pathological basis of all cancers, potential targets for therapy and treatment protocols employed to improve the management of cancer patients. The journal also focuses on the impact of management programs and new therapeutic agents and protocols on

\section{Dovepress}

patient perspectives such as quality of life, adherence and satisfaction. The manuscript management system is completely online and includes a very quick and fair peer-review system, which is all easy to use. Visit http://www.dovepress.com/testimonials.php to read real quotes from published authors. 